\title{
Algumas considerações a respeito da alternância verbal na aquisição do português brasileiro
}

\author{
Carla Pereira Minello \\ Universidade Estadual de Campinas (UNICAMP), Campinas, São Paulo, Brasil \\ carlaminello@gmail.com
}

DOI: http://dx.doi.org/10.21165/el.v45i2.718

\begin{abstract}
Resumo
Neste artigo, propõe-se uma discussão sobre o fenômeno da alternância causativo-incoativa na aquisição do português brasileiro por meio de dados de produção espontânea. Toma-se como ponto de partida o fato de que na forma anticausativa é possível recuperar um causador implícito, ao contrário do que acontece nas sentenças inacusativas, o que evidencia estruturas diferentes para essas duas construções. Toma-se, como hipótese, que a criança, no processo de aquisição da linguagem, é sensível à diferença existente entre as anticausativas e os inacusativos.
\end{abstract}

Palavras-chave: aquisição da linguagem; alternância verbal; anticausativo; inacusativo; português brasileiro.

\section{Some Considerations about Verbal Alternation in the Acquisition of Brazilian Portuguese}

\begin{abstract}
This paper discusses the causative-inchoative alternation in the acquisition of Brazilian Portuguese by examining data that contain childrens' spontaneous production. The starting point of this article is that it is possible to identify an implicit causer in anti-causatives, which is not possible in unaccusatives. This fact reveals that there are different structures for these constructions. The hypothesis for this study is that children is able to perceive the difference between anticausative and unaccusative structures in the language acquisition process.
\end{abstract}

Keywords: language acquisition; verbal alternation; anticausatives; unaccusatives; Brazilian Portuguese.

\section{Introdução}

A alternância causativo-incoativa é um fenômeno caracterizado por verbos que apresentam uma forma transitiva, como ilustrada em (1a) e uma forma anticausativa, forma decorrente da alternância causativo-incoativa, ilustrada em $(1 b)^{1}$.
a. A Maria quebrou o vaso
b. O vaso quebrou

A alternância causativo-incoativa não é permitida com todo verbo transitivo, como pode ser observado em (2).

\footnotetext{
${ }^{1}$ Há uma variedade na terminologia ligada ao fenômeno da alternância verbal de verbos causativos: alternância causativa, alternância ergativa, alternância transitiva, entre outros. Para os propósitos deste trabalho, será adotada a terminologia alternância causativa-incoativa (SCHER et al., 2009). O mesmo vale para a forma intransitiva, que será nes te trabalho tratada como anticausativa (ALEXIADOU et al., 2006).
} 
As sentenças transitivas que permitem que ocorra alternância causativo-incoativa são aquelas em que o verbo na sua forma transitiva é um verbo causativo. Verbos causativos licenciam todo tipo de argumento externo, como um agente (3a), causador (3b), evento-causador (3c) e instrumento (3d) (ALEXIADOU et al., 2006).

(3)
a. O João quebrou a janela
b. A tempestade quebrou a janela
c. As marteladas do João quebraram a janela
d. A pedra quebrou a janela / Eu quebrei a janela com uma pedra

Outra restrição é que as anticausativas ocorrem apenas com determinados argumentos internos:

(4) a. He broke his promise / the contract / the world record

Ele quebrou sua promessa / o contrato / o recorde mundial

b. *His promise / *the contract / *the world record broke

*Sua promessa /*o contrato / *o recorde mundial quebrou

(5) a. He broke the vase / Ele quebrou o vaso

b. The vase broke / $O$ vaso quebrou

Como pode ser observado inicialmente, não são todos os verbos transitivos que sofrem alternância causativo-incoativa e essa ocorre apenas com determinados tipos de argumento interno. Nas seções seguintes serão apresentados argumentos que distanciam sentenças inacusativas de sentenças anticausativas e que colocam a última mais próxima às sentenças passivas, apresentando qual a proposta adotada por este trabalho e, por fim, serão apresentados dados preliminares de aquisição, trazendo algumas considerações.

\section{Do causativo ao anticausativo}

As análises canônicas para alternância causativo-incoativa propõem que a forma anticausativa não possui um argumento externo implícito, em comparação com sentenças passivas, como $O$ vaso foi quebrado, em que há um argumento externo implícito (ALEXIADOU et al., 2006). Uma dessas análises pressupõe uma regra lexical de causativização, em que a forma causativa (transitiva) é derivada da forma anticausativa (DOWTY, 1979).

A segunda proposta considera a forma causativa do verbo como a forma básica, e a forma anticausativa derivada desta, em que a perda do argumento externo ocorre via regra lexical de detransitivização (LEVIN; RAPPAPORT-HOVAV, 1995; REINHART, 2000). Na detransitivização existiria uma forma verbal transitiva primitiva que, por meio da aplicação de uma regra lexical, levaria à perda de qualquer argumento externo implícito, resultando em um afastamento entre as anticausativas e a passiva verbal. Evidências usadas para justificar uma provável detransitivização estão relacionadas a distinções entre passivas e anticausativas presentes no inglês: sentenças passivas podem ser modificadas por by-phrases e advérbios orientados para o agente e permitem controle em sentenças adverbiais de finalidade (purpose clauses), enquanto que esses processos 
não são permitidos nas anticausativas, conforme ilustrado nas sentenças de (6) a (8) ${ }^{2}$ (ALEXIADOU et al, 2006).

(6) a. The boat was sunk by Bill / O barco foi afundado pelo Bill

b. *The boat sank by Bill / *O barco afundou pelo Bill

a. The boat was sunk on purpose / O barco foi afundado de propósito

b. *The boat sank on purpose / ?O barco afundou de propósito

a. The boat was sunk to collect the insurance / O barco foi afundado para coletar o seguro

b. *The boat sank to collect the insurance / ? O barco afundou para coletar o seguro

Uma terceira proposta, mais recente (ALEXIADOU et al., 2006), não parte de regras lexicais mas assume que duas formas alternantes não estão em uma relação derivacional, ou seja, nem a forma intransitiva derivaria da forma transitiva e nem forma transitiva derivaria da forma intransitiva. As autoras defendem que as evidências contra a causativização e contra a detransitivização são encontradas nas diferenças translinguísticas relacionadas aos argumentos externos de passivas e de sentenças incoativas e ao conjunto de verbos que se submetem à alternância.

As evidências apontadas por Alexiadou e Anagnostopoulou (1999; 2004; 2007) e Alexiadou et al. (2006), contrárias à proposta da detransitivização, apontam a possibilidade de o inglês licenciar um causador ou um evento-causador quando estes são introduzidos pela preposição from, conforme ilustrado em (9). Já o alemão licencia o uso da preposição durch quando o argumento externo é um causador ou um evento-causador tanto em construções passivas (10a e 11a), quanto em anticausativas (10b e 11b) (ALEXIADOU et al., 2006).

(9) a. The window cracked/broke from the pressure / A janela rachou/quebrou pela pressão

b. The window cracked/broke from the explosion / A janela rachou/quebrou pela explosão

c. *The door opened from Mary/from the key / *A janela abriu pela Mary/chave

(10) a. Die Vase wurde durch den Erdstoß zerbrochen

The vase was through-the earth tremor broken

The vase was broken by the earth tremor / O vaso foi quebrado pelo tremor de terra

b. Die Vase zerbrach durch ein Erdbeben

The vase broke through an earthquake / O vaso quebrou através de um terremoto

(11) a. Die Luftqualität im Raum wird durch das Rauchen von Zigaretten verschlechtert

The air-quality in-the room is through the smoking of cigarettes worsened

The air quality in the room is worsened by the smoking of cigarettes / A qualidade do arna sala é agravada pela fumaça dos cigarros

b. Die Luftqualität im Raum verschlechtert sich $d u r c h$ das Rauchen von Zigaretten massiv

The air-quality in-the room worsens REFL through the smoking of cigarettes severely

Pode ser observado nos exemplos de (9) a (11) que o funcionamento das anticausativas no inglês e no alemão se assemelham, não licenciando um agente em

\footnotetext{
${ }^{2}$ As sentenças (7b) e (8b), em português, são aceitas de forma aparentemente não-sistemática por alguns falantes de português do estado de São Paulo. Para alguns desses falantes, (7b) é indeterminada e a (8b) tem sentido passivo.
} 
construções anticausativas, mas permitindo um causador ou um evento causador, o que é evidenciado pelo uso das preposições from para o inglês e durch para o alemão. Outra língua em que as anticausativas se comportam de forma semelhante ao já descrito é o grego, que não licencia um agente introduzido pela preposição apo em construções anticausativas como em (12a), mas licencia um causador introduzido pelas preposições apo e me (12b) ou um evento-causador introduzido pela preposição me $(12 \mathrm{c})^{3}$. No grego é possível também o licenciamento de um instrumento introduzido pela preposição $m e$, como exemplificado em (12d) (ALEXIADOU et al., 2006).

a. *Ta mallia mu stegnosan apo tin komotria

The hair my dried-Act by the hairdresser

* My hair bried by the hairdresser

b. Ta ruxa stegnosan apo/me ton ilio

The clothes dried-Act by/with the sun

*The clothes dried by the sun

c.Ta ruxa stegnosan me to aploma ston ilio

The clothes dried-Act with the hanging-up under the sun

*The clothes dried by hanging them up under the sun

d. Ta mallia mu stegnosan $m e$ to pistolaki

The hair my dried-Act with the hair-dryer

* My hair dried with the hair dryer

Para os autores, a gramaticalidade das sentenças (9a), (9b), (10), (11a), (11b), (12b), (12c) e (12d) indicam a presença de um causador implícito nas anticausativas. A distribuição das preposições from ou durch nas passivas e nas anticausativas evidenciam a presença de diferentes tipos de núcleos Voice e do núcleo CAUS nas duas sentenças, assim como a distribuição das preposições apo e me nas anticausativas do grego evidenciariam a presença desses dois núcleos, tal como argumentado em Alexiadou et al. (2006).

Aproximar as anticausativas das passivas com base na presença do núcleo Voz e do núcleo CAUS leva a considerar que a projeção $v \mathrm{P}$ dessas construções seja estruturalmente diferente da projeção $v \mathrm{P}$ de verbos tais como os inacusativos. A seguir, será apresentada a proposta adotada por este trabalho, em que se assume CAUS como uma propriedade e não como um núcleo, conforme apresentado anteriormente.

\section{Entre o inacusativo e o anticausativo}

Na seção anterior foram apontadas evidências de que as anticausativas apresentam um comportamento sintático semelhante ao das sentenças passivas. A seguir, serão mostrados argumentos que distanciam as anticausativas dos verbos inacusativos.

\footnotetext{
${ }^{3}$ No grego, o uso das preposições apo e me nas anticausativas são licenciados quando introduzem um causador ou um evento-causador, exemplificado em (16). As anticausativas não licenciam agentes introduzidos pela preposição apo, mas licenciam causadores e evento-causador.
} 
A proposta que será adotada neste trabalho tem como base Scher et al. (2009), em que se discute a estrutura argumental de verbos no PB. Os autores partem dos pressupostos de que os papéis dos argumentos são aspectuais e que esses são definidos em relação aos núcleos funcionais ou às raízes dentro de $v P$, sendo que essas raízes são acategóricas e licenciam determinadas estruturas de evento; os verbalizadores seriam três: estados (BE), incoativos (GO) e atividades e eventos; $e$ as derivações se dariam por fases (SCHER et al., 2009, p. 185-186).

A assunção dos pressupostos listados acima levou os autores a considerar a presença ou ausência de duas propriedades dentro da estrutura de um evento: DIN e CAUS. A primeira dirá se a raiz se associa a uma eventualidade dinâmica; a segunda, se a raiz se associa a uma eventualidade causada. Além disso, a presença ou ausência da propriedade CAUS dirá sobre a concatenação da raiz à um DP ou diretamente ao $v$.

Por exemplo, verbos transitivos que não permitem alternância. Esses verbos teriam uma raiz dinâmica, mas não causada, a qual denotaria os traços [+DIN, -CAUS], em que a raiz se combina diretamente com o $v$ e o DP complemento se combina com o nó do vezinho, diferentemente do que ocorre na estrutura de verbos que sofrem alternância. A estrutura para esse tipo de verbo apresentada em Scher et al. (2009) está reproduzida em (13).

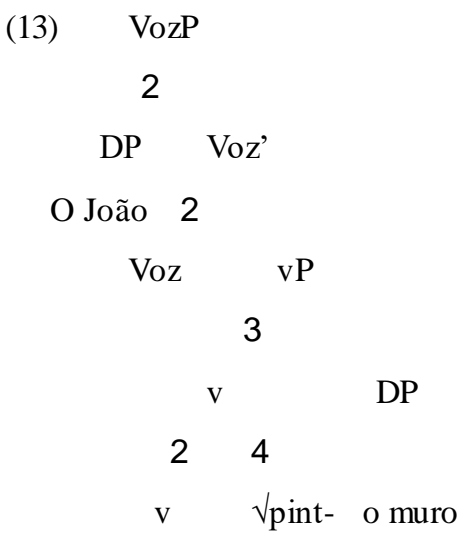

De acordo com Scher et al. (2009), os verbos que sofrem alternância causativoincoativa possuiriam uma estrutura em que apresentariam uma forma causativa "na qual um vezinho introduz um evento causador, não especificado e o sintagma raiz introduz outra eventualidade, estativa ou dinâmica, que é causada" (SCHER et al., 2009, p. 187). Essa estrutura seria bieventiva e a raiz do verbo se combinaria diretamente com o DP complemento, sendo que (i) a raiz do verbo denotaria um estado e teria a matriz de traços [-DIN, +CAUS] ou (ii) um evento dinâmico seria denotado pela raiz, sendo que a matriz de traços associada à raiz é [+DIN, +CAUS] (SCHER et al., 2009). Em (14) está representada a estrutura sintática para o verbo abrir proposta em Scher et al. (2009).

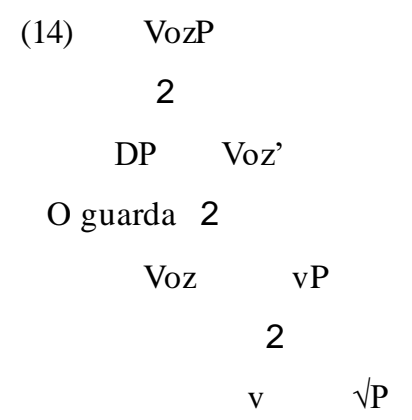




$$
\text { DP } 2 \text { abr- }
$$

A porta

A estrutura sintática proposta para as anticausativas em (14) se diferenciaria da estrutura proposta para os verbos que são exclusivamente inacusativos, como, por exemplo, nascer, chegar. A estrutura em (14) não serviria para representar verbos inacusativos, pois se esperaria uma transitivização dos inacusativos (SCHER et al., 2009), o que não acontece, como observado em (15).

$$
\begin{aligned}
& \text { a. A carta chegou } \\
& \text { b. *O João chegou a carta. }
\end{aligned}
$$

Scher et al. (2009) assume que em verbos exclusivamente inacusativos haveria um vezinho diferente daquele presente em outras estruturas, como nos verbos de alternância causativo-incoativa, e que, portanto, este tipo de verbo não poderia ser causativisado. Para captar essa diferença, os autores apresentam a estrutura reproduzida em (16), que difere da estrutura apresentada em (14).

(16)

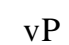

2

$\mathrm{DP} \quad \sqrt{\mathrm{P}}$

O João 2

vGO $\sqrt{ }$ cheg-

Segundo os autores, o $v$ dos inacusativos ${ }^{4}$ seria incoativo, diferente do $v$ eventivo dos verbos transitivos e, por tal motivo, não poderia ser causativizado, sendo que a raiz seria [+DIN, -CAUS]. Esse $v$ introduz uma eventualidade incoativa, não permitindo anexar um núcleo Voz na estrutura e, sem a anexação desse núcleo, a posição de especificador não seria criada, o que impossibilitaria os verbos inacusativos de sofrerem alternância causativo-incoativa ou permitirem o uso do clítico se, como apresentado abaixo.

Uma evidência de que há diferenças entre a camada $v \mathrm{Pde}$ sentenças anticausativas e de sentenças inacusativas, ilustrada de (17) a (19) é o uso obrigatório do clítico se em línguas como o francês e o espanhol e o uso optativo desse clítico no PB nas sentenças anticausativas, assim como a agramaticalidade das sentenças inacusativas com esse clítico.
a. La ventana se cerró
b. La fenêtre s'est fermée
c. A janela (se) fechou
a. El vaso se rompió
b. Le verre s'est cassé
c. O vaso (se) quebrou

\footnotetext{
${ }^{4}$ Scher et al. (2009) apontam que as propostas existentes até o momento não conseguem dar conta dos verbos inacus ativos que não estão envolvidos emestruturas de alternância causativo-incoativa e que mes mo a estrutura por eles apresentada não considera algumas características dos verbos inacusativos, as quais não serão discutidas neste artigo.
} 

a. * La carta se llegó / La carta llegó
b.*La lettre s'est arrivée / La lettre est arrivée
c.*A carta se chegou / A carta chegou

A partir do que foi discutido até o momento, adota-se a hipótese de que o mecanismo sintático envolvido na alternância causativo-incoativa, que resulta em sentenças como $O$ copo quebrou ou $O$ pneu furou, é diferente do mecanismo sintático que leva a existir estruturas com verbos inacusativos como A carta chegou.

\section{A alternância causativo-incoativa na aquisição do português: uma breve discussão}

\section{O corpus observado}

Foram analisados dados de fala espontânea de 59 arquivos de 4 crianças das bases do Projeto Aquisição da Linguagem (CEDAE, IEL/UNICAMP) e do Banco de Dados do CEAAL (Centro de Aquisição e Aprendizagem de Linguagem/PUCRS), entre um ano e seis meses a cinco anos e seis meses, conforme disposto no quadro 1.

Quadro 1: Sessões analisadas

\begin{tabular}{|l|c|}
\hline \multicolumn{1}{|c|}{ Criança e Idade } & Número de sess ões \\
\hline R. (entre 1 ano e 5 meses a 4 anos e 10 meses) & 31 sessões \\
\hline V. (entre 3 anos e 2 meses a 5 anos e 6 meses) & 15 sessões \\
\hline AC. (entre 1 ano e 10 meses a 3 anos e 7 meses) & 7 sessões \\
\hline G. (entre 1 ano e 10 meses a 3 anos e 6 meses) & 6 sessões \\
\hline
\end{tabular}

Foram observadas as ocorrências de verbos inacusativos com argumento interno realizado por DP (20a e 20b), pronominal (20c) e as ocorrências de verbos transitivos em sua forma de alternância $D P V$ (20d). Sentenças transitivas do tipo $V D P$ não foram observadas por não ser possível saber que interpretação a criança deu para esse tipo de ocorrência.
a. Caiu papóca. (pipoca) (R., 1 ano, 7 meses e 21 dias)
b. A perua chego logo (V., 4 anos, 10 meses e 25 dias )
c. Ele caiu. (G., 2 anos, 3 meses e 17 dias)
d. Meu macacão lavou (V., 3 anos, 7 meses e 19 dias)

\section{Apresentando os dados}

Foram obtidas 211 ocorrências de verbos inacusativos a partir dos dados de produção espontânea observados, sendo que em 128 o argumento interno era nulo, em 31 ele foi realizado com pronome e em 52, realizado com DP. Para o propósito deste trabalho, as ocorrências em que o argumento era nulo não foram consideradas.

As 31 ocorrências do argumento interno realizado com um pronome se deram todas na ordem $D P V$, como em (21).
a. Ele caiu. (G., 2 anos, 3 meses e 17 dias)
b. Olha onde ele caiu, ó! (R., 3 anos, 4 meses e 9 dias)
c. Ah... como ela caiu? (V., 3 anos, 4 meses e 18 dias) 
d. Acho que ela caiu no meu colo. (AC., 3 anos, 7 meses e 6 dias)

Das 52 ocorrências em que o argumento interno foi realizado com DP, 34 eram argumentos internos pós-verbais, como em (22) e em dezoito o argumento interno era pré-verbal, como exemplificado em (23).

(22) a. Caiu papóca. (pipoca) (R., 1 ano, 7 meses e 21 dias)

b. Chegou a máquina de lavar. (G., 3 anos e 21 dias)
a. O trem vai chegar (G., 2 anos, 3 meses e 17 dias)
b. A Jéssica caiu (R., 3 anos, 10 meses e 2 dias)
c. Quase que a poltrona caiu (V., 4 anos, 3 meses e 15 dias)
d. A perua chego logo (V., 4 anos, 10 meses e 25 dias)

As ocorrências do DP interno em posição pós-verbal iniciaram-se expressivamente por volta dos três anos. No entanto, já ocorreram antes dessa idade, como pode ser visto em (24a), enquanto que o aparecimento do DP interno em posição pré-verbal tornou-se mais expressivo após os três anos de idade, com poucas ocorrências de inacusativos com DP pré-verbal antes dos três anos (24a). Isto pode sugerir que a posição default do argumento interno dos verbos inacusativos no PB é a pós-verbal.

As ocorrências de anticausativas nos dados observados foram cinco, apresentadas em (24).
a. A aga abiu (R., 2 anos e 12 dias)
b. Só que a rodinha da do skate estagou (AC., 3 anos e 29 dias)
c. Meu macacão lavou (V., 3 anos, 7 meses e 19 dias)
d. A igrejinha fechou (V., 4 anos, 10 meses e 6 dias)
e. O sol fechou (V., 4 anos, 10 meses e 6 dias)

Há dúvidas sobre se as sentenças (24a), (24d) e (24e) são ocorrências de anticausativas. A sentença (24a) deixa dúvidas sobre qual seria sua natureza e as sentenças $(24 \mathrm{~d})$ e $(24 \mathrm{e})$ aparentam ser ecos de uma música previamente aprendida pela criança.

\section{Discussão}

$\mathrm{Na}$ literatura existente sobre aquisição de inacusativos (25a), voz passiva (25b) e estrutura de alçamento (25c) existe a hipótese de que essas estruturas são de aquisição mais tardia pela criança, pois os mecanismos sintáticos envolvidos na formação dessas sentenças ainda não estariam adquiridos, uma vez que estariam sujeitos à maturação (WEXLER, 2004).
a. A carta chegou
b. A carta foi enviada
c. O rapaz parece que enviou a carta

Para Wexler (2004), o mecanismo envolvido na formação de sentenças como as representadas em (25) é o movimento do DP para uma posição mais alta, sendo que até a idade de cinco anos, a criança não seria capaz de realizar esse tipo de movimento. O autor, 
para justificar esse aparente atraso na aquisição de sentenças como em (25), se apoia na Condição de Impenetrabilidade de Fase ${ }^{5}$, proposta por Chomsky (2001).

De acordo com Chomsky (2001), $v \mathrm{P}$ e CP são fases e apenas suas margens (núcleos e especificadores) ficam visíveis para a próxima fase, enquanto que seu domínio interno não, tão logo que a derivação é enviada para Spell Out. No caso dos verbos transitivos $v \mathrm{P}$ é uma fase e no momento que a derivação é enviada para Spell Out, o domínio interno VP não está mais visível para operações, como movimento. Já nos verbos inacusativos, nas construções passivas e nas estruturas de alçamento, $v \mathrm{P}$ não é uma fase. Isto leva a, por exemplo, o complemento de VP permanecer visível para operações futuras, como o movimento do DP complemento para uma posição mais alta, SpecTP. De acordo com Wexler (2004), a criança até os cinco anos de idade trataria o $v \mathrm{P}$ dos verbos inacusativos, das passivas e das estruturas de alçamento como sendo fase, tornando, dessa forma, impossível o movimento do DP complemento de VP para a posição de SpecTP, o que resultaria na agramaticalidade destas construções.

A proposta de Wexler (2004) considera que os inacusativos, a voz passiva e as estruturas de alçamento teriam um $v$ do mesmo tipo, defectivo e que a aparente dificuldade da criança reside em tratar esse $v$ como não defectivo, impossibilitando $o$ movimento do DP complemento. Ao contrário do que propõe Wexler (2004), este trabalho está assumindo que o $v$ de verbos inacusativos seria incoativo e não permita a projeção de um especificador, por não ser possível anexar o núcleo funcional Voz acima de $v \mathrm{P}$, uma vez que a única eventualidade presente é incoativa. Já o $v$ dos anticausativos introduz uma eventualidade causadora, em que esse evento se identifica com o evento introduzido pelo núcleo Voz.

Os achados encontrados por meio da produção espontânea infantil sinalizam que a criança é sensível às diferenças existentes entre as construções (23) e (24), mas é necessário que essa hipótese seja testada experimentalmente. Evidências de que a criança é sensível à diferença existente entre A carta chegou e A porta abriu se deve ao aparecimento precoce de inacusativos em dados de produção espontânea e ao aparecimento mais tardio de anticausativas, uma vez que os mecanismos sintáticos e semânticos envolvidos nas anticausativas possuem uma maior complexidade que aqueles envolvidos nos inacusativos.

\section{Algumas consi derações}

Observou-se que a ocorrência de verbos inacusativos na fala de criança falante do PB inicia-se antes dos dois anos, sendo que as primeiras ocorrências desse verbo com argumento interno realizado foram registradas com um ano e sete meses de idade. A ocorrência de verbos transitivos com e sem argumento realizado se deu a partir dos dois anos de idade.

Tendo como ponto de partida que as anticausativas, assim como as passivas, possuem a estrutura de $v \mathrm{P}$ diferente da estrutura do $v \mathrm{P}$ dos verbos inacusativos e de que anticausativas e voz passiva não se comportariam como os verbos inacusativos, não

5 Condição de Impenetrabilidade de Fase ou Phase Impenetrability Condition (PIC): $O$ domínio de H não está acessível a operações fora de HP; apenas $H$ e sua margem estão acessíveis para tais operações (CHOMSKY, 2001, p. 13, tradução própria). 
assumo a hipótese de que o movimento do objeto para o especificador de TP seja um fator relevante para a aquisição de anticausativas.

A aparente intransitividade em sentenças como Fechou a porta / A porta fechou e Quebrou a janela / A janela quebrou se dá muito mais superficialmente do que derivacionalmente. Uma evidência é a possibilidade da inserção do clítico se nessas estruturas em francês, espanhol e português e a distribuição das preposições em inglês e alemão, como foi apresentado na introdução deste trabalho, o que sinaliza uma estrutura sintática diferente entre as anticausativas e os inacusativos.

Por fim, uma abordagem não-derivacional aparenta ser mais interessante para o tratamento das anticausativas por possibilitar uma maior aproximação entre essas sentenças e sentenças passivas e um maior distanciamento do tratamento desses dois fenômenos como sendo intransitivos.

\section{REFERÊNCIAS}

ALEXIADOU, A.; ANAGNOSTOPOULOU, E. Non-active morphology and the direction of transitivity alternations. In Proceedings of NELS 29, Vol. II, p. 27-40, 1999.

Voice morphology in the causative inchoative alternation: evidence for a nonunified structural analysis of unaccusatives. In: ALEXIADOU, A. et al. (orgs.). The Unaccusativity Puzzle: Explorations of the Syntax-Lexicon Interface. New York: Oxford University Press, 2004. p. 114-136.

ALEXIADOU, A.; ANAGNOSTOPOULOU, E.; SCHÄFER, F. The properties of Anticausatives crosslinguistically. In: RIEMSDIJK, H. et al. (orgs). Phases of Interpretation. Berlim: Mouton de Gruyter, 2006. p. 184-211.

ALEXIADOU, A.; ANAGNOSTOPOULOU, E. Agent, Causer and Instrument PPs in Greek: implications for verbal structure. In: Workshop on Greek Syntax and Semantics MIT 20-22.5. 2007. MIT Working papers, 2007, s/p.

ALEXIADOU, A. On the morphosyntax of (anti-)causative verbs. In: RAPPAPORT, M et al. (orgs). Syntax, Lexical Semantics, and Event Structure. New York: Oxford University Press, 2010. p. 177-203.

CHOMSKY, N. Derivation by phase. In: KENSTOWICZ, M. (org). Ken Hale: A Life in Language. Cambridge: MIT Press, 2001. p. 1-52.

DOWTY, D. Word Meaning and Montague Grammar: The Semantics of Verbs and Times in Generative Semantics and in Montague's Ptq. Amsterdan: Springer Netherlands, 1979. 418p.

HIRSCH, C.; WEXLER, K. Children's Passives and Their Resulting Interpretation. In: DEEN, J. et al. (orgs). The Proceedings of the Inaugural Conference on Generative Approaches to Language Acquisition-North America. Connecticut: UConn Occasional Papers in Linguistics, 2006. p. 5-36.

HOVAV, M. R.; LEVIN, B. Lexicon uniformity and the causative alternation. In: SILONI, T. et al. (orgs). The Theta System: Argument Structure at the Interface. New York: Oxford University Press, 2011. p. 150-176. 
LEVIN, B.; RAPPAPORT-HOVAV, M. R. Unaccusativity: At the Syntax-Lexical Semantics Interface. Cambridge: MIT Press, 1995. 336 p.

SCHER, A. P.; MEDEIROS, A. B.; MINUSSI, R. D. Estrutura argumental em morfolo gia distribuída. In: NAVES, R. R. et al. (orgs). Estudos formais da gramática das línguas naturais. Goiânia: Cânone Editorial, 2009. p. 123-138.

TSIMPLI, I. M. The acquisition of voice and transitivity alternations in Greek as native and second language. In: UNSWORTH, S. et al. (orgs.). Paths of Development in L1 and L2 Acquisition: In Honor of Bonnie D. Scwartz. Language Acquisition and Language Disorders. Amsterdan: John Benjamins Publishing Company, 2006. p. 15-55.

WEXLER, K. Theory of Phasal Development: Perfection in Child Grammar. In: CSIRMAZ, A. et al. (orgs). Plato's Problems: Papers on Language Acquisition. Cambridge: MIT Working Papers in Linguistics, 2004. p. 159-209.

Recebido em: 04/10/2015

Aprovado em: 02/05/2016 